\title{
Confronting the fissions and fusions of human fertilization
}

\author{
David F. Albertini
}

Published online: 26 April 2014

(C) Springer Science+Business Media New York 2014

Two year ago, an article appeared in JARG documenting and confirming for the first time a fast block to polyspermy in the human (Mio et al., Possible mechanism of polyspermy block in human oocytes observed by time-lapse cinematography, 2012 J Assist Reprod Genet, 29:951-956, DOI 10.1007/ s10815-012-9815). That the fusion of sperm and egg plasma membranes launches the program of development from which organisms arise remains a central tenet in plant and animal biology. And, as has been appreciated for many years, the ability of the oocyte to fend off supernumerary sperm and assure a diploid composition of chromosomes in the resulting zygote relies on the establishment of a block to polyspermy through mechanisms that we now understand to be of both fast and slow varieties. While the existence of fast and slow blocks to polyspermy has been well documented in divergent metazoan organisms, controversy remained as to the presence of a fast block in the human oocyte - that is, until the publication of Mio and colleagues - mainly because of the lack of imaging strategies permitting the tracking multiple sperm during the process of in vitro fertilization (IVF). Even though the problems associated with polyspermy persist in the practice of human ARTs, and the introduction of ICSI in the 1990s has greatly reduced the incidence of this type of genetic imbalance during the generation of human embryos, it remains a serious problem for clinics that continue to use IVF in their daily practices. Most scientists and clinicians would argue for the continuation of investigations into the basic mechanisms underscoring human fertilization even in the face

Capsule Human fertilization viewed in the context of the molecules that foster sperm and fusion proffers explanations for clinical failures in human ARTs but also prompts debate on the impact of reproductive science in a broader realm of human health.

D. F. Albertini $(\bowtie)$

University of Kansas Medical Center, Kansas, KS, USA

e-mail: dalbertini@kumc.edu of opposition from governments and societies uncomfortable with such lines of research.

At the heart of all such studies has been the lingering fascination with the various fissions and fusions featured at the level of the plasma membrane of gametes. Whether your fancy be the acrosome reaction, intermingling of sperm and egg membranes, or the burst of exocytosis of cortical granules that stages the so-called slow block to polyspermy, the precision and celerity of membrane dynamics that defines successful fertilization stands alone as one of the most spectacular biological phenomena with which we remain obsessed - and for good reason!

So important was the discovery of the molecular machinery that guides membranes to and through their appointments with fission and fusion that it was recognized as worthy of the 2013 Nobel Prize in Physiology and Medicine. It turns out, and not so surprisingly, that proteins have evolved for the expressed purpose of consummating the intimate contacts made between a variety of apposed membranes (be they of cells or their internal organelles) effecting true fusion. Viruses have been known for years to deploy such molecules, also known as fusogens, to invade cells and more in line with the world of reproductive medicine are fusogens believed to participate in the formation of syncytial trophoblast. A central candidate by the name of SYNCYTIN 1 is the gene product now widely suspected to mediate this aspect of placental development in the human and other species.

This month, JARG features a series of papers pertaining to male infertility and sperm physiology. At the top of our list is the paper by Bjerregaard and colleagues from Copenhagen entitled "Syncytin-1 and its receptor is present in human gametes" (10.1007/s10815-014-0224-1). This work alerts our readership generally, and those fertilization aficionados here and beyond, to the demonstration of plausible functional cooperativity for the fusion of human sperm and eggs. Drawing upon the sensitivity qRT-PCR, both sperm specific 
expression of SYNCYTIN 1 and oocyte-specific expression of the syncytin receptor ASCT-2 mRNAs are documented, positioning these candidate fusion partners in a gender-specific context with clear implications for human ARTs. Moreover, the paper goes on to show a SYNCYTIN 1 pattern of protein expression in the equatorial segment of acrosome reacted sperm, the specialized domain of the sperm plasma membrane long recognized to be the site of sperm-egg fusion (see this month's cover). What findings like this will mean to the daily practice of human ARTs and our understanding of failures in fertilization will require further experimentation and analysis. And, lest we forget, the identification of molecules mediating sperm-egg fusion may spark a new wave of contraceptive development research upon which even larger problems in reproductive health could be confronted for the overall benefit of humankind.

Besides focusing on issues of male reproductive health this month, we are offering a template for discourse on the importance of reproductive science and medicine in the larger arena of scientific publishing. A study conducted by Duncan, Woodruff, and colleagues at Northwestern University prompts this topic with the following question: why is it that important papers in the field of reproduction rarely appear in the pages of the top journals? (Duncan et al., A small field for fertile science: the low visibility of reproductive science in high impact journals, 10.1007/s10815-014-0205-4 ). It comes as no great surprise that part of the answer derives from our small stature relative to other disciplines. And who could ignore the vestigial Victorian undercurrents that some societies have retained effectively thwarting advances in the fields of embryonic stem cells and regenerative medicine, as we have recognized in past issues of JARG. This paper, and the accompanying editorial (Woodruff et al., Keeping reproductive science visible, viable, and valuable - a call to rethink how we publish, 10.1007/s10815-014-0206-3), are timely contributions that we hope will prompt debate in the coming months, and we welcome your comments and insights into this matter.
Along these lines, it is important to keep in mind what leaders in the scientific enterprise globally are perceiving for the future of scientific publishing. It is therefore of interest to note that last December, the day before accepting the 2013 Nobel prize in Physiology and Medicine, Randy Schekman from the University of California San Francisco aired his sentiments regarding the influence of high impact journals on the perceptions and practice of science. His commentary, entitled "How journals like Nature, Cell and Science are damaging science," draws parallels between the dark side of incentivizing on the banking and finance industries and the practices top journals commonly deploy to advance themselves and the authors whose work bears their trademarks (www.theguardian.com/commentisfree/2013/dec/09/howjournals-nature-science-cell-damage-science). Schekman's message draws upon what he believes has transformed the foundations of scientific integrity accorded to "discovery" into a business model rife with spurious and misleading commercial interests. From their lofty perch, he notes that these journals use the impact factor as more of a "gimmick" than a metric worthy of adoption in achieving academic rank or obtaining grants, a reliance that far too many promotion committees and funding agencies have become reliant upon for making big decisions for forging ahead within the purview of the biomedical research enterprise. He argues further that more and more of the very best publications are finding their way into open access journals and that this medium may return the field of scientific publication to a level of legitimacy it so deserves.

Maybe our shared fascinations with the building blocks of human reproduction need a little more interdisciplinary fission, fusion, and recycling before moving discovery into the arena of translational medicine. Journals continue to influence funding agendas, academic advancement, and therapeutic direction for better or worse. The time has arrived for reproductive science to assume a larger identity that separates impact blurred by commercial interests or egos from the laudable goal of improving reproductive health for everyone. 